\title{
Cancellation of Crosstalk-Induced Jitter
}

\author{
James F. Buckwalter, Student Member, IEEE, and Ali Hajimiri, Member, IEEE
}

\begin{abstract}
A novel jitter equalization circuit is presented that addresses crosstalk-induced jitter in high-speed serial links. A simple model of electromagnetic coupling demonstrates the generation of crosstalk-induced jitter. The analysis highlights unique aspects of crosstalk-induced jitter that differ from far-end crosstalk. The model is used to predict the crosstalk-induced jitter in 2-PAM and 4-PAM, which is compared to measurement. Furthermore, the model suggests an equalizer that compensates for the data-induced electromagnetic coupling between adjacent links and is suitable for pre- or post-emphasis schemes. The circuits are implemented using 130-nm MOSFETs and operate at 5-10 Gb/s. The results demonstrate reduced deterministic jitter and lower bit-error rate (BER). At $10 \mathrm{~Gb} / \mathrm{s}$, the crosstalk-induced jitter equalizer opens the eye at $10^{-12}$ BER from 17 to 45 ps and lowers the rms jitter from 8.7 to $6.3 \mathrm{ps}$.
\end{abstract}

Index Terms-Bounded-uncorrelated jitter, deterministic jitter, equalization, high-speed serial link, jitter.

\section{INTRODUCTION}

B ROADBAND communication links face many challenges as speeds move into microwave frequencies. In particular, dense communication environments, required to satisfy demand for high aggregate throughput, need special consideration to meet future data rates. At high frequencies, serial links are susceptible to electromagnetic interference as well as strong attenuation and reflections caused by poor electrical interfaces.

Backplane interconnects are particularly limited at higher frequencies. These interconnects suffer from attenuation due to skin losses, via stubs, and connectors, severely limiting the signal integrity [1], [2]. To combat high-frequency attenuation, amplitude equalization is introduced in the transmitter (pre-emphasis) or receiver (post-emphasis) [3], [4]. Equalizer implementation is straightforward at the receiver, but high-frequency attenuation requires amplification of the signal and, therefore, the noise, limiting the signal-to-noise ratio (SNR) [5]. Pre-emphasis compensates high-frequency attenuation prior to the addition of noise over the interconnect. This approach, however, suffers drawbacks in environments where several serial links are situated in close proximity. In backplanes as well as integrated circuit environments, a premium on space precludes creating completely shielded links. Therefore, as we move to faster data rates, high-frequency signal components couple more electromagnetic energy into neighboring channels [3]. This coupling manifests as near-end crosstalk (NEXT) and far-end crosstalk (FEXT) [6].

Crosstalk results from the interaction of electromagnetic fields generated by neighboring data signals as they propagate

Manuscript received June 30, 2005; revised September 15, 2005. This work was supported by the National Science Foundation and an IBM Ph.D. Fellowship.

The authors are with the California Institute of Technology, Pasadena, CA 91125 USA (e-mail: jamesb@ caltech.edu).

Digital Object Identifier 10.1109/JSSC.2005.864113

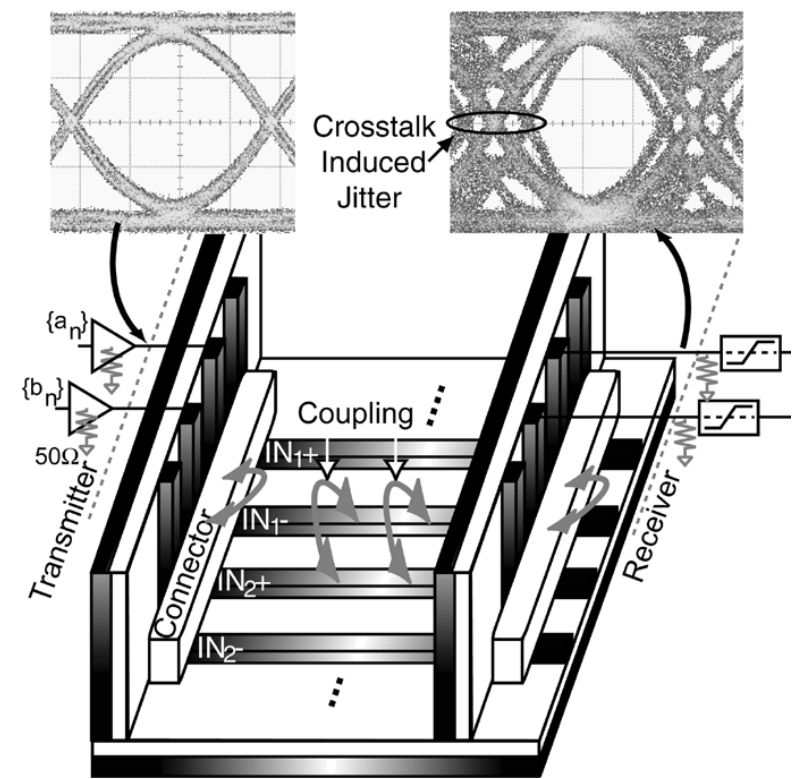

Fig. 1. Crosstalk jitter generated in the data eye due to the influence of a neighboring signal.

through transmission lines and connectors, as shown in Fig. 1. A review of crosstalk mechanisms in different backplane and on-chip interconnections is offered in [7]. For instance, wiring capacitances tend to dominate interconnect crosstalk on-chip and backplane connectors introduce multi-pin crosstalk. Recent work has addressed crosstalk amplitude equalization issues between neighboring serial links [8]-[10]. Crosstalk considerations also affect the performance of integrated digital circuits [11]. However, no effort to date has been made to equalize the timing jitter resulting from crosstalk.

When signal transitions occur, high-frequency energy couples between adjacent wires. At the receiver, the timing of both data eyes is obscured. This timing deviation due to FEXT is called crosstalk-induced jitter (CIJ). While FEXT causes CIJ, CIJ behaves differently than FEXT for digital modulation schemes. In particular, CIJ is insensitive to signal swing and rise time.

This paper reviews crosstalk in signals on coupled lines in Section II. The impact of crosstalk on jitter is described in Section III. The electromagnetic mode between data transitions induces this jitter. This discussion expands on earlier descriptions in [12]. CIJ is a type of bounded-uncorrelated jitter (BUJ), a subset of deterministic jitter (DJ) [13], [14].

DJ reduces the sampling range of the data eye, degrading the bit-error rate (BER) [15]. In Section IV, we apply the model to CIJ in $M$-level pulse amplitude modulation (PAM), of which 2-PAM and 4-PAM are conventionally implemented, to illustrate the jitter tradeoffs associated with higher order modulation schemes. 
Reducing DJ is important to designing robust links with low BER. In Section V, we propose general equalizer implementations for minimizing the impact of CIJ. The CIJ equalizer determines the electromagnetic modes of transitions and adjusts the delay of each transition. These equalizers rely on dynamic adjustment of the transition time of the data signal and not amplitude emphasis. The circuit implementation in a 130-nm MOS technology is discussed in Section VI [16]. Finally, the performance of a CIJ equalizer is presented in Section VII in terms of eye opening and BER improvement.

\section{CRosstalk in Transmission Lines}

In modern high-speed communication links, data are transmitted across parallel transmission lines [5]. The mutual capacitance and inductance induces fluctuations on adjacent transmission lines. This impairment is referred to as crosstalk.

A lumped-element model for the transmission lines demonstrates the effect of coupled electromagnetic energy in Fig. 2. The model consists of the self capacitance and inductance. The coupling occurs through the mutual capacitance, $C_{m}$, and the mutual inductance, $L_{m}$. The telegraphist's equations describe the spatial and temporal variation of voltage and current on the adjacent lines [17].

$$
\begin{aligned}
\frac{\partial}{\partial z}\left[\begin{array}{l}
v_{1} \\
v_{2}
\end{array}\right] & =-\left[\begin{array}{cc}
L_{s} & L_{m} \\
L_{m} & L_{s}
\end{array}\right] \frac{\partial}{\partial t}\left[\begin{array}{l}
i_{1} \\
i_{2}
\end{array}\right] \\
\frac{\partial}{\partial z}\left[\begin{array}{l}
i_{1} \\
i_{2}
\end{array}\right] & =-\left[\begin{array}{cc}
C_{s} & -C_{m} \\
-C_{m} & C_{s}
\end{array}\right] \frac{\partial}{\partial t}\left[\begin{array}{l}
v_{1} \\
v_{2}
\end{array}\right] .
\end{aligned}
$$

Solutions to this problem are eigenmodes associated with an even and odd mode. The even mode is excited when $v_{1}(x, t)=$ $v_{2}(x, t)$ and the odd mode is excited when $v_{1}(x, t)=-v_{2}(x, t)$. Any arbitrary excitation is represented through a linear combination of these two modes. For data communications, we are concerned primarily with three modes: the even, odd, and superposition when no data transition occurs. We illustrate these three situations in Fig. 3.

The data transitions occur independently on adjacent lines and, therefore, the mode changes randomly. Consequently, we want to demonstrate the variation in the propagation time for transitions on the lines.

\section{A. Time of Flight}

The impact of the coupling mode on data transitions is intuitively approached by calculating the time of flight (TOF). TOF is determined from the odd and even mode propagation constants for the eigenmodes. From [18], the propagation constants are

$$
\begin{aligned}
& \beta_{o}=\omega \sqrt{\left(L_{s}-L_{m}\right)\left(C_{s}+C_{m}\right)} \\
& \beta_{e}=\omega \sqrt{\left(L_{s}+L_{m}\right)\left(C_{s}-C_{m}\right)} .
\end{aligned}
$$

The relationship between the propagation constant and the phase velocity, $v_{p}=\omega / \beta$, determines the TOF. Consequently, the odd and even TOF are

$$
\begin{aligned}
& T_{o}=l \sqrt{\left(L_{s}-L_{m}\right)\left(C_{s}+C_{m}\right)} \\
& T_{e}=l \sqrt{\left(L_{s}+L_{m}\right)\left(C_{s}-C_{m}\right)} .
\end{aligned}
$$

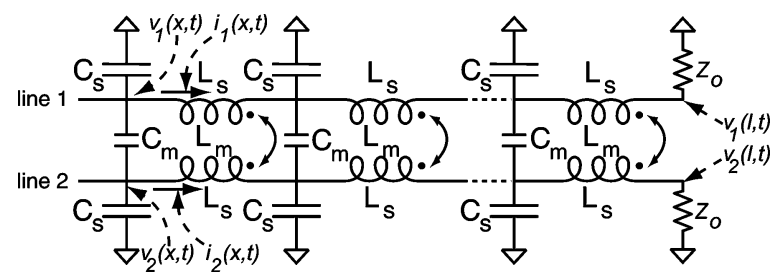

Fig. 2. Lossless coupled transmission line model consisting of the self capacitance and inductance and mutual capacitance and inductance.

\section{Odd Mode Superposition Mode Even Mode}

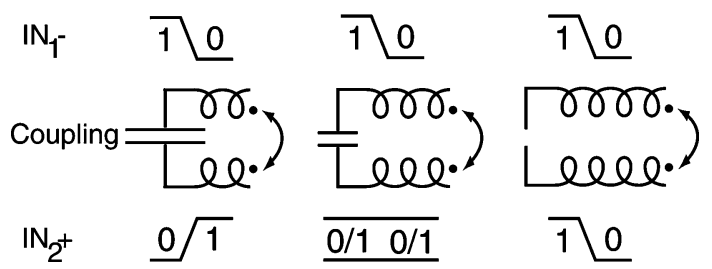

Fig. 3. Modes that occur between the coupled transmission line.

As expected, the TOF increases with the length $l$ of the line. While the odd and even mode TOFs differ, the superposition mode is not readily determined due to the nature of pulse propagation down the line. As an edge propagates down the transmission line, it induces a voltage spike on the quiet adjacent line. This spike, in turn, propagates with the edge and grows in amplitude. Eventually, the spike will, in turn, influence the arrival time of the original edge.

Clearly, the speed of the two modes are equal if $L_{m} / L_{s}=C_{m} / C_{s}$. This condition is guaranteed when the transmission line is homogeneous. Homogeneity holds in stripline transmission lines because of field symmetry. For microstrip lines, homogeneity is not generally guaranteed since the electric and magnetic fields above and below the line are not symmetric [17]. In general, interconnects are often implemented as microstrip lines and are inhomogeneous.

To determine the superposition mode arrival time, the FEXT generated between two transmission lines is solved in Appendix A. This derivation relies on a weak coupling argument to allow a Taylor series approximation of the modes [18]. The TOF for the superposition mode is thus expressed as $T=l \sqrt{L_{s} C_{s}-L_{m} C_{m}} \sim l \sqrt{L_{s} C_{s}}$. Consequently, the even and odd mode TOF demonstrated in (3) are treated as perturbations from this value.

$$
T_{e(o)} \approx T \pm \frac{l}{2}\left(\frac{L_{m}}{Z_{o}}-C_{m} Z_{o}\right)
$$

where the even mode is the addition and the odd mode is the difference. The perturbation in (4) can be expressed as a forward coupling time constant, $\tau_{\mathrm{f}}$, defined as

$$
\tau_{f}=\frac{l}{2}\left(C_{m} Z_{o}-\frac{L_{m}}{Z_{o}}\right) .
$$

The forward coupling time constant behavior depends on the implementation. For high impedance lines, the capacitance dominates and $\tau_{f} \approx l C_{m} Z_{o} / 2$ [11], [17]. In this case, the odd 
mode is slower than the even mode. For low impedance lines, the inductance dominates and $\tau_{f} \approx-l L_{m} /\left(2 Z_{o}\right)$. In this case, the arrival time of the even and odd modes reverse.

\section{CROSSTALK-INDUCED JITTER}

While crosstalk-induced jitter is caused by FEXT, the behaviors differs from FEXT in several ways. In this section, the TOFs of the even, odd, and superposition mode are incorporated into a model for data communications to define crosstalk-induced jitter (CIJ). Discussions and measurements of CIJ support these results.

Often, high-speed data are transmitted differentially. Between the differential lines, a virtual ground exists. Depending on the relative intra-pair and inter-pair spacing of the differential lines, CIJ can be dominated by the effect of one adjacent data signal. The jitter on $\mathrm{IN}_{1}+$ and $\mathrm{IN}_{1}-$ might be generated by different neighbors. To simplify the analysis, we study coupling between $\mathrm{IN}_{1}-$ and $\mathrm{IN}_{2}+$. However, more sophisticated modeling can analyze the effect over a larger number of neighbors.

If adjacent transitions occur simultaneously, we can develop a time domain representation for the induced jitter. However, even with some timing skew between lines, the derivation of CIJ can still be applied as will be discussed. If we label $r_{1}(t)$ the victim signal and $r_{2}(t)$ the aggressor signal, these signals can be represented as

$$
\begin{aligned}
& r_{1}(t)=\sum_{n=-\infty}^{0} a_{n} g(t-n T) \\
& r_{2}(t)=\sum_{n=-\infty}^{0} b_{n} g(t-n T)
\end{aligned}
$$

where $a_{n}$ and $b_{n}$ are independent data sequences and $g(t)$ is the pulse response of the channel, which is assumed to be identical on both lines. Following the derivation in [12], we calculate the arrival time of the victim signal at the decision threshold. When there is no aggressor present, $v_{t h}=r_{1}\left(t_{c}\right)$ and variations of the threshold crossing time $t_{c}$ are related to other sources of jitter (i.e., random and deterministic jitter). When the aggressor signal is present, the coupling introduces the data from the aggressor to the victim. The time constant derived in the previous section defines a high-pass filter and couples the high-frequency components of the aggressor data. Therefore, the arrival time in the presence of crosstalk, $t_{x}$, is found from the solution of this algebraic equation:

$$
r_{1}\left(t_{x}\right)+\tau_{f} \frac{d}{d t} r_{2}\left(t_{x}\right)=v_{t h}
$$

In Appendix B, suitable approximations are presented to solve this equation. The result is that the variation of the threshold crossing time due to CIJ is

$$
t_{c, C I J}=t_{x}-t_{c}=-\tau_{f} \cdot \frac{b_{0}-b_{-1}}{a_{0}-a_{-1}} .
$$

The denominator represents the transition in the victim data and the numerator represents the transition in the aggressor data. Note that $(8)$ is only valid when a transition occurs on the victim data, i.e., $a_{0} \neq a_{-1}$.
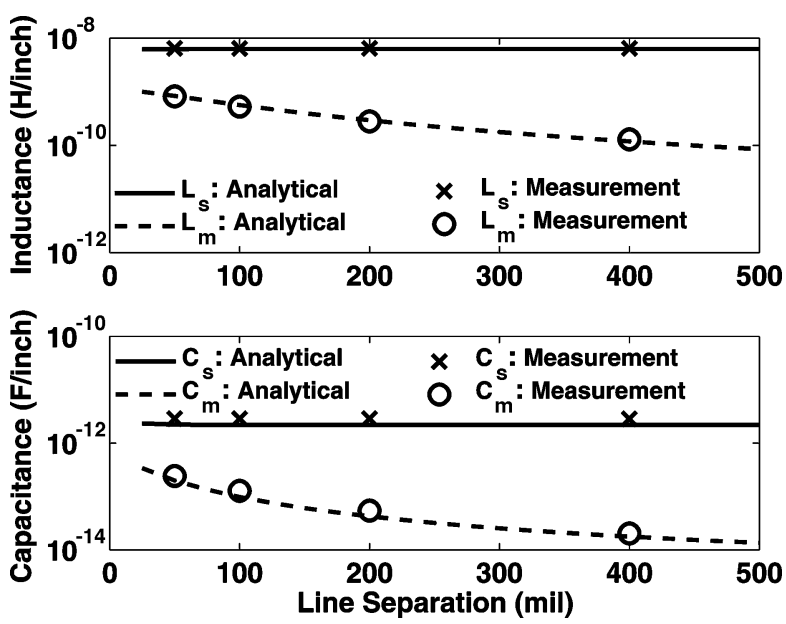

Fig. 4. Comparison of calculated and measured inductances and capacitances for coupled microstrip lines on Rogers 5880.

Most importantly, the details of $g(t)$ are not apparent in (8). This is an interesting result because it highlights the differences between CIJ and FEXT voltage noise. For FEXT, slower transitions generate less crosstalk-induced noise on the victim line [5]. However, in terms of jitter, the victim edges are also slow and more susceptible to jitter. Therefore, the CIJ is not sensitive (to the first order) to the transition slope.

Additionally, the amplitudes of $a_{n}$ and $b_{n}$ cancel out of the ratio of the aggressor and victim data. This implies that the CIJ is independent of signal swing. Therefore, pre-emphasis does not significantly impact CIJ and cannot be used to improve it.

To verify these expressions for CIJ, a set of 8-inch singleended microstrip lines are constructed with Rogers 5880 duroid $\left(\varepsilon_{\mathrm{r}}=3.3\right)$. The dielectric thickness was $62.5 \mathrm{mil}$ and each $50-\Omega$ transmission line was 200 mil wide. The coupled lines are designed with impedance matched to the superposition mode. The microstrip lines are separated by $50 \mathrm{mil}, 100 \mathrm{mil}, 200 \mathrm{mil}$, and 400 mil. The capacitance and inductance of the lines is calculated analytically from formulas presented in [19] and [20]. These calculated self inductance and capacitance and mutual inductance and capacitance are measured experimentally with an LCR meter. The inductance and capacitances match well at each of the line separations as shown in Fig. 4.

The TOF of the even and odd modes is calculated from the extracted parameters in Fig. 5. ADS transient simulations are also used to calculate the mode TOF. The superposition time of flight normalizes the plot so that the results reflect the differential TOF. The plot demonstrates that the TOF increases with decreasing line spacing. Furthermore, the odd mode travels faster than the even mode over the transmission lines, indicating that the inductance is the dominant coupling effect for the transmission lines.

\section{A. Effect of Timing Offset Between Victim and Aggressor}

While thus far the analysis assumes isosynchronous signal transmission over the interconnect, CIJ is relatively insensitive to timing offsets between the victim and the aggressor.

Examining our analysis for CIJ in Appendix A, a phase offset could be added in the numerator or denominator. Notably, the 


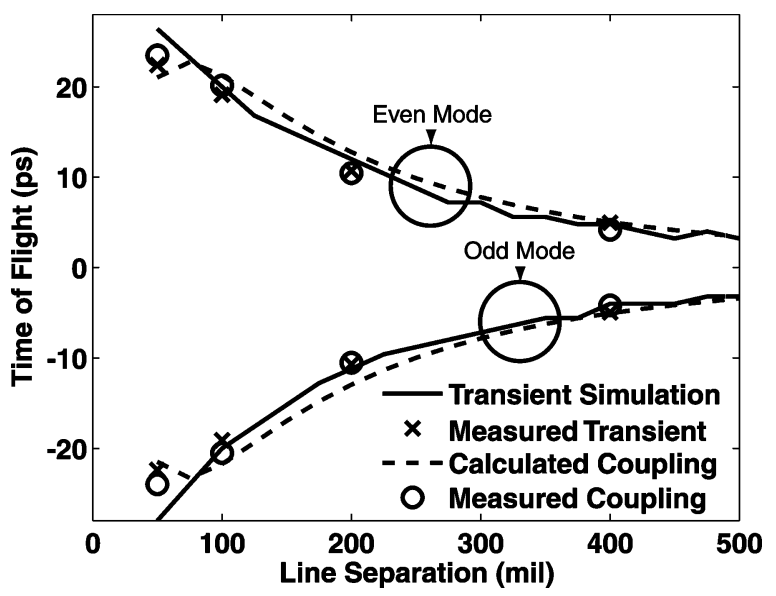

Fig. 5. Time of flight for the microstrip lines.

slope of $g(t)$ in the numerator and denominator cancels out. At small timing offsets, the slope is constant and (8) still holds. Basically, we expect that the range of time when the crosstalk translates into CIJ follows the rise and fall time of the signal slope.

For larger phase offsets, the crosstalk occurs in the data eye and has little effect on the transition timing. Consequently, there is a phase offset at which CIJ becomes an amplitude ISI problem.

In Fig. 6, the jitter is recorded as a function of the offset between the victim and aggressor signals. Both the peak-to-peak and rms jitter are recorded. Additionally, the arrival time of the even and odd modes is plotted on the right axis. Two features are striking. First, the CIJ is pronounced after a small offset range. Second, the peak-to-peak and rms jitter are essentially constant over a large range of offsets. A small minimum occurs in the rms jitter when the even and odd mode arrival times are equally spaced from the superposition mode.

Notably, in many backplane applications, the skew specifications for adjacent channels are much larger than the rise and fall time of the signals. Consequently, it is difficult to predict whether FEXT between a pair of adjacent signals will result in CIJ.

\section{CRosstalk Jitter IN $M$-PAM}

Recent papers have proposed the use of 4-PAM to overcome channel limitations and we can show the jitter issues of 4-PAM [4], [8], [21]. In this section, the CIJ expression is applied to 2-PAM and 4-PAM schemes and we develop expressions for rms and peak-to-peak expressions for the jitter based on uncoded data statistics. These expressions are useful in comparing 2-PAM and 4-PAM in terms of the CIJ penalty.

\section{A. 2-PAM}

For binary data $\left(a_{n}=\{0,1\}\right)$, the ratio in (8) can assume only three different values: $-1,0$, and 1 . Thus, the coupling spreads the transition time of the victim data (and, symmetrically, the aggressor data) between three discrete values as shown in the original eye in Fig. 1. The probability density function is

$$
\operatorname{pdf}_{\mathrm{CIJ}}\left(t_{c}\right)=\frac{1}{4} \delta\left(t_{c}+\tau_{f}\right)+\frac{1}{2} \delta\left(t_{c}\right)+\frac{1}{4} \delta\left(t_{c}-\tau_{f}\right) .
$$

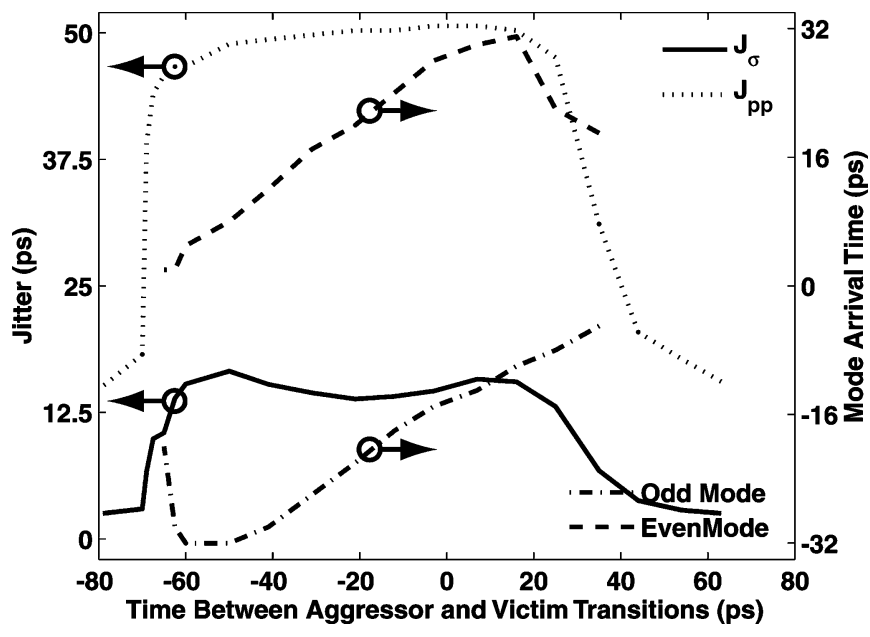

Fig. 6. Timing offset between the aggressor and victim data at $5 \mathrm{~Gb} / \mathrm{s}$ does not dramatically affect the rms and peak-to-peak jitter.

The middle, unaltered data transition occurs twice as frequently because the superposition mode occurs twice as often as either the odd or even modes. With this pdf, we calculate the BER penalty resulting from the crosstalk.

The rms value CIJ, $J_{\sigma, \text { CIJ }}$, is determined from the pdf in (9). The rms value is useful for determining the timing margins that will achieve a particular BER [13].

$$
J_{\sigma, \mathrm{CIJ}}=\frac{\tau_{f}}{\sqrt{2}} .
$$

Additionally, the peak-to-peak jitter, $J_{\mathrm{pp}, \mathrm{CIJ}}$, is calculated from the bounds of this discrete pdf. The peak-to-peak jitter gives the total range over which transitions occur. Note that the rms jitter is always smaller than the peak-to-peak jitter.

$$
J_{\mathrm{pp}, \mathrm{CIJ}}=2 \tau_{f}
$$

In Fig. 7, a 10-Gb/s data eye is demonstrated for two line separations. These eyes illustrate the three distinct peaks and increased separation between the peaks for narrow separations.

The jitter statistics are calculated for each of the line separations. Each data eye is recorded with and without the effect of the aggressor to normalize for other sources of jitter. This correction for the rms and pp jitter is

$$
\begin{aligned}
J_{\sigma, \mathrm{CIJ}} & =\sqrt{J_{\sigma, \text { meas,agg }}^{2}-J_{\sigma, \text { meas,no_agg }}^{2}} \\
J_{\mathrm{pp}, \mathrm{CIJ}} & =J_{\mathrm{pp}, \text { meas,agg }}-J_{\mathrm{pp}, \text { meas,no_agg }} .
\end{aligned}
$$

Statistics are recorded from a histogram recorded with the Agilent 81600B wide-bandwidth oscilloscope. The vertical opening of the histogram is $1 \mathrm{mV}$ and we record 5000 data points in the histogram. The rms jitter is recorded from the histogram and we use (10) to calculate the forward coupling time constant and compare it with the results in Fig. 5. The statistics are presented in Table I for all four line separations. $J_{\sigma, \text { CIJ }}$ increases with decreasing line separation. Additionally, the time constants associated with the rms and pp jitter are similar and provide reasonable agreement with the results of Fig. 5. 


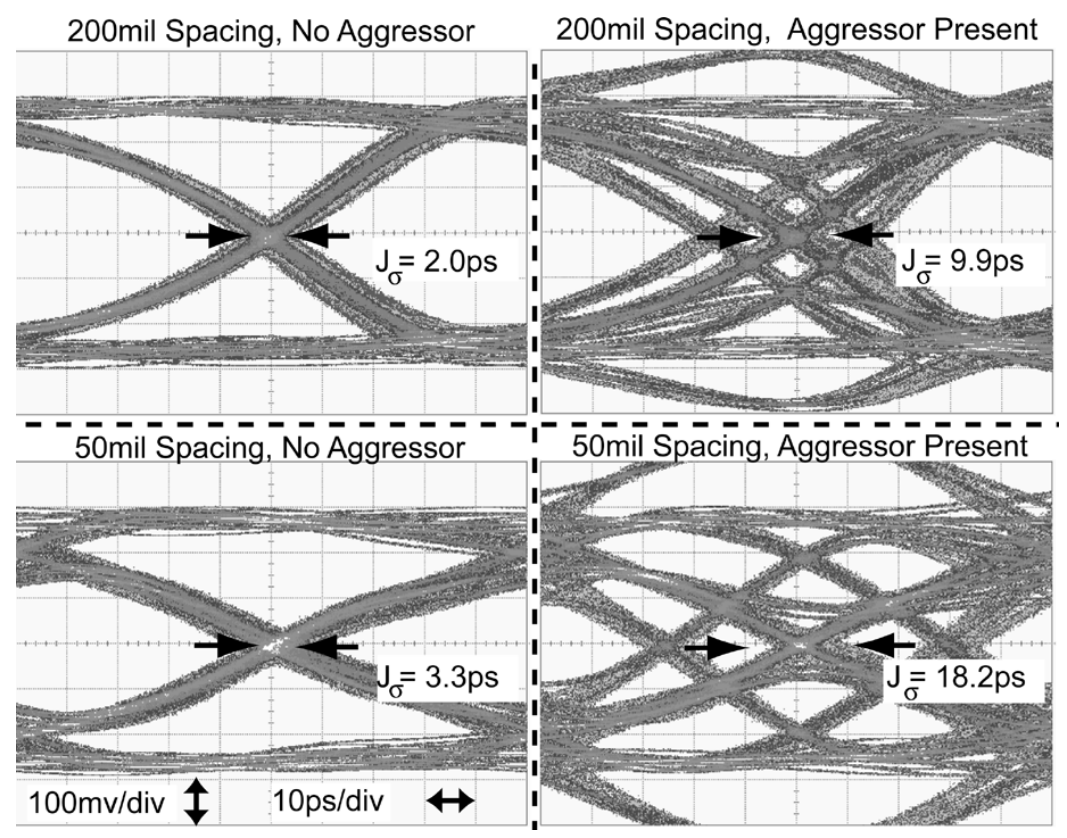

Fig. 7. 2-PAM data eyes at $10 \mathrm{~Gb} / \mathrm{s}$ shown with and without the aggressor.

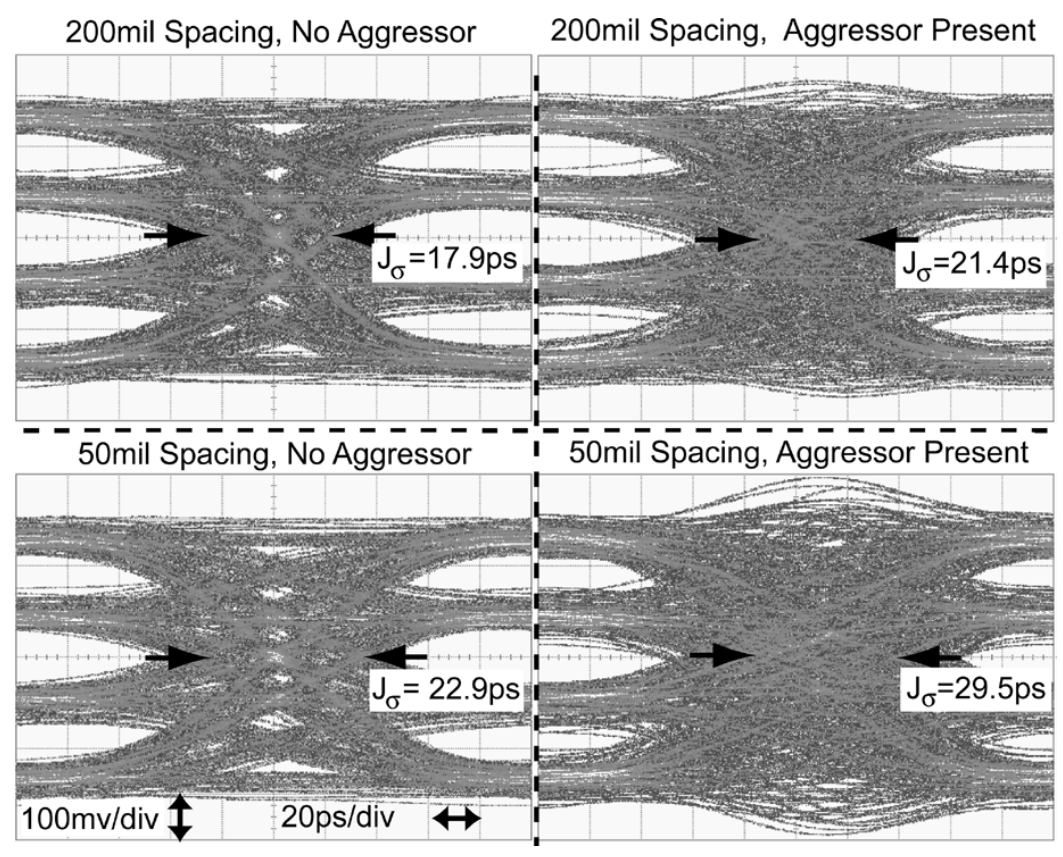

Fig. 8. 4-PAM data eyes at $5 \mathrm{GS} / \mathrm{s}(10 \mathrm{~Gb} / \mathrm{s})$ with and without aggressor.

\section{B. 4-PAM}

When equally probable quaternary signaling is employed $\left(a_{n}=\{0,1,2,3\}\right)$, the analysis of jitter is more sophisticated. The larger signal swings imply that stronger aggressive signals can couple into weaker victim transitions. Additionally, if the symbols are equally probable, the transition density of 4-PAM is $75 \%$, which is higher than the transition density for 2-PAM. The multiple transitions make 4-PAM CIJ more problematic.

Examining the ratio in (8), we anticipate that the CIJ in 4-PAM results in fifteen different threshold crossing deviations, significantly more than the three calculated for 2-PAM. In Appendix C, we demonstrate the pdf for 4-PAM CIJ. A variety of definitions are possible for the jitter of the 4-PAM data eye
TABLE I

CROSSTALK-INDUCED JITTER IN 2-PAM

\begin{tabular}{|c|c|c|c|c|}
\hline Line Separation & $400 \mathrm{mil}$ & $200 \mathrm{mil}$ & $100 \mathrm{mil}$ & $50 \mathrm{mil}$ \\
\hline $\mathrm{J}_{\sigma}$, No Aggressor & $2.5 \mathrm{ps}$ & $2.0 \mathrm{ps}$ & $2.3 \mathrm{ps}$ & $3.3 \mathrm{ps}$ \\
\hline $\mathrm{J}_{\sigma}$, Aggressor & $5.4 \mathrm{ps}$ & $9.9 \mathrm{ps}$ & $14.5 \mathrm{ps}$ & $18.2 \mathrm{ps}$ \\
\hline $\mathrm{J}_{\sigma, \mathrm{CIJ}}$ & $4.7 \mathrm{ps}$ & $9.7 \mathrm{ps}$ & $14.3 \mathrm{ps}$ & $17.9 \mathrm{ps}$ \\
\hline Extracted $\tau_{\mathrm{f}}$ & $6.6 \mathrm{ps}$ & $13.7 \mathrm{ps}$ & $20.2 \mathrm{ps}$ & $25.3 \mathrm{ps}$ \\
\hline
\end{tabular}

and we use only the central voltage threshold to calculate the CIJ pdf. To summarize the results of the Appendix, the rms jitter for 4-PAM is 
TABLE II

CROSSTALK-INDUCED JITTER IN 4-PAM

\begin{tabular}{|c|c|c|c|c|}
\hline Line Separation & $400 \mathrm{mil}$ & $200 \mathrm{mil}$ & $100 \mathrm{mil}$ & $50 \mathrm{mil}$ \\
\hline $\mathrm{J}_{\sigma}$, No Aggressor & $16.4 \mathrm{ps}$ & $17.9 \mathrm{ps}$ & $20.6 \mathrm{ps}$ & $22.9 \mathrm{ps}$ \\
\hline $\mathrm{J}_{\sigma}$, Aggressor & $17.6 \mathrm{ps}$ & $21.4 \mathrm{ps}$ & $25.5 \mathrm{ps}$ & $29.5 \mathrm{ps}$ \\
\hline $\mathrm{J}_{\sigma, \mathrm{CIJ}}$ & $6.4 \mathrm{ps}$ & $11.6 \mathrm{ps}$ & $15.0 \mathrm{ps}$ & $18.5 \mathrm{ps}$ \\
\hline Extracted $\tau_{\mathrm{f}}$ & $6.4 \mathrm{ps}$ & $11.6 \mathrm{ps}$ & $15.0 \mathrm{ps}$ & $18.5 \mathrm{ps}$ \\
\hline
\end{tabular}

In Appendix C, we demonstrate the pdf for 4-PAM. A variety of definitions are discussed in this Appendix regarding the precise calculation of rms jitter value. To summarize the results of the Appendix, the rms CIJ, $J_{\sigma, 4 \text {-PAM }}$ is

$$
J_{\sigma, \mathrm{CIJ}}=\tau_{f} \sqrt{\frac{145}{144}} \approx \tau_{f}
$$

A direct comparison with the CIJ calculated for 2-PAM demonstrates that the rms jitter for 4-PAM is $40 \%$ greater than for 2-PAM. The effect of crosstalk is demonstrated in Fig. 8 and shows the ambiguity introduced into the timing deviations of the data eye due to the aggressive signal. The result for each of the four line separations is shown in Table II. The measurements are used to calculate the forward coupling time constant from (13). The rms jitter, however, does not agree as closely for close line separations due to the additional contributions of data-dependent jitter.

\section{CRosstalk-Induced JitTer Equalization}

To minimize the BER in the serial link, we want to avoid the timing ambiguity introduced by CIJ. In the previous sections, we developed an analysis of crosstalk-induced jitter and pdf for the threshold crossing time. The separation of discrete peaks is related to the strength of the coupling between the two lines and the signaling scheme.

Therefore, we suggest an equalization scheme for links where CIJ severely degrades the BER. A general analog scheme for a CIJ equalizer for 2-PAM is proposed in Fig. 9. A symbol period time delay $T$ is used to capture the data values before and after the transition. The summing device computes the difference between these data values to produce a three-level signal corresponding to whether the current transition is a rising or falling edge. This result is multiplied by the result of the neighboring line to resolve the transition mode. The resulting tri-state signal indicates the electromagnetic mode of the transitions. For example, if both the victim and aggressor data lines have a rising (or falling) edge, the multiplication results in one, indicating the even mode. The tri-state value associated with the detection of this mode is weighted by an appropriate crosstalk equalization coefficient, which adjusts the time delay of the transition. This shifts timing deviations that occur in (9) to a consistent transition edge (i.e., the central transition).

Notably, this adjustment can be implemented in either the transmitter as a pre-emphasis technique or the receiver before detection of the signal value.

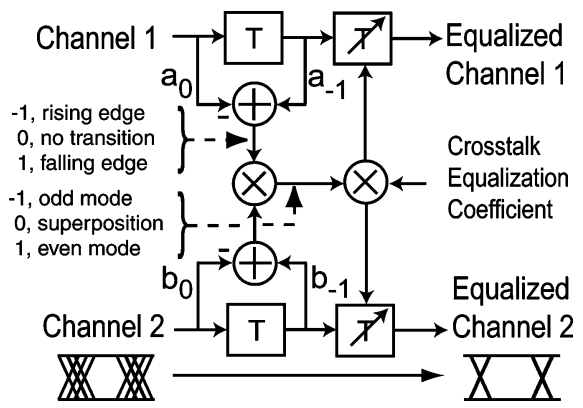

Fig. 9. Schematic of a two-channel crosstalk-induced jitter equalization.

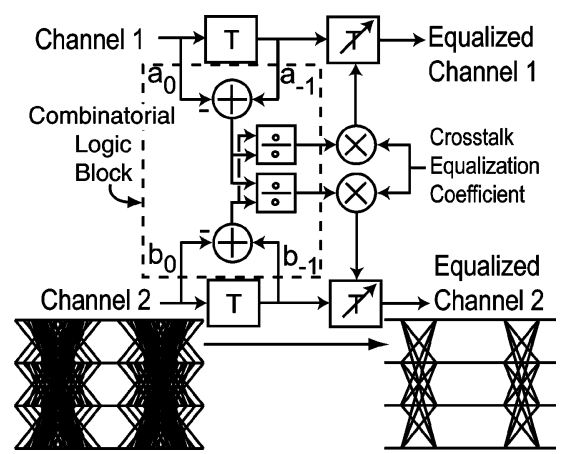

Fig. 10. Schematic of a two-channel 4-PAM crosstalk-induced jitter equalization.

The algorithm demonstrated in Fig. 9 exactly compensates the effect of CIJ. The compensation introduces a time delay, $t_{\text {comp }}$, to both channels equal to

$$
t_{\mathrm{comp}}=\tau_{f}\left(b_{0}-b_{-1}\right)\left(a_{0}-a_{-1}\right) .
$$

When this is added to the deviation occurring due to the CIJ, we have a total timing deviation of

$$
t_{c, \mathrm{CIJ}}+t_{\mathrm{comp}}=\tau_{f}\left(b_{0}-b_{-1}\right)\left[-\frac{1}{\left(a_{0}-a_{-1}\right)}+\left(a_{0}-a_{1}\right)\right] .
$$

Therefore, this CIJ equalizer operates correctly as long as $\left|a_{0}-a_{-1}\right|=1$. Therefore, this scheme will not function properly for 4-PAM schemes. The advantage of the proposed circuit in Fig. 9 is its ease of adaptation to high-speed mixed-signal implementation. Additionally, the circuit in Fig. 9 can be extended to multiple neighbors with a straightforward adaptation of the demonstrated algorithm. The mode detection can be calculated between additional neighbors if necessary. Expanding this scheme will come at the cost of additional circuit and wiring complexity to route the mode detection signal between other neighboring links.

A hypothetical CIJ equalizer for 4-PAM is suggested in Fig. 10. In this schematic, we have used a division element to compute the voltage ratio in (8). Two calculations are required because the compensation is different for each channel. In particular, the channels are reciprocally related and, therefore, the computational symmetry of CIJ in 2-PAM does not hold. 


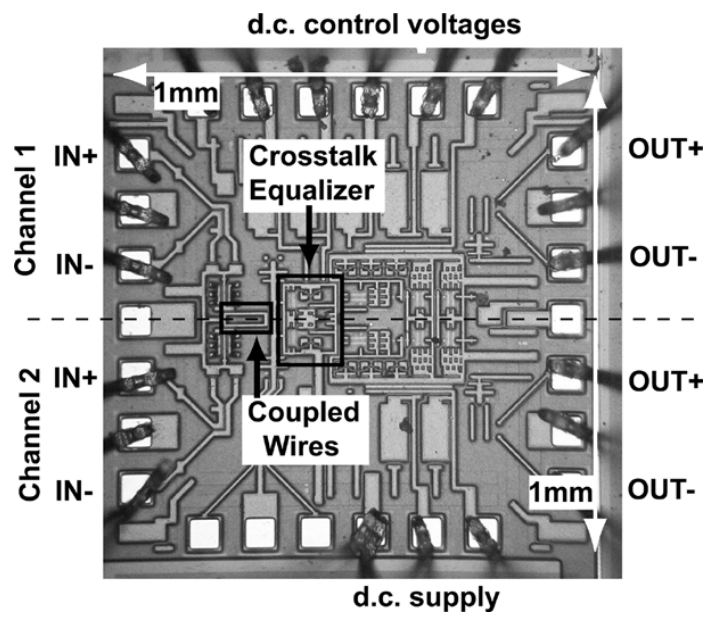

Fig. 11. Chip microphotograph of the crosstalk-induced jitter equalizer.

This proposed CIJ equalizer for 4-PAM compensates all the possible crosstalk-induced timing deviations.

In principle, this equalizer circuit could be implemented in high-speed links. However, the challenge is to maintain low complexity and, consequently, low power consumption. One approach is not to attempt to compensate all of the 15 threshold crossing time deviations, as the schematic does, but to reduce the problem to compensating only critical deviations. For instance, the victim signal is most severely impacted when the data transition is small $\left(\left|a_{0}-a_{-1}\right|=1\right)$ and the aggressor signal swing is large $\left(\left|b_{0}-b_{-1}\right|=2,3\right)$. Compensating these conditions would correct for the largest timing deviations, $2 \tau_{\mathrm{f}}$ and $3 \tau_{\mathrm{f}}$. Notably, analog implementations of FIR filters have been the best solutions for 4-PAM crosstalk cancellation [8]-[10].

\section{CirCuit ImPlementations}

The circuit is implemented with 130-nm MOSFETs using the IBM 8HP process. A chip microphotograph is provided in Fig. 11 and includes the CIJ equalizer as well as an on-chip coupled wire section for testing the circuit operation. These are described in more detail in Section VII. The circuit implementation focuses on compensating CIJ between two channels using 2-PAM. It consists of high-speed current-mode logic (CML) exclusive-or (XOR) and AND gates to detect transitions and compare the mode at $10 \mathrm{~Gb} / \mathrm{s}$ as illustrated in Fig. 12. While analog implementations of the circuits in Fig. 9 are possible, this logic gate approach is more robust to process variations and is sufficient for a proof-of-concept of the jitter equalizer.

The logic gates generate logical values when the even or odd modes occur and are multiplied by a coefficient to adjust a variable time delay. An XOR detects a transition on each line. The results of the neighboring line are ANDed to determine whether either the even or odd mode has occurred. An XOR between each line determines which mode is present. Since the timing adjustment in this case is feed-forward, the delay of the logic gates can be compensated by adding delay in the data paths.

The multiplexer combines the results of the even and odd transition detection to adjust the time delay. The multiplexer circuit diagram and operation is illustrated in Fig. 13. Three output voltages are generated that depend on the differential control of

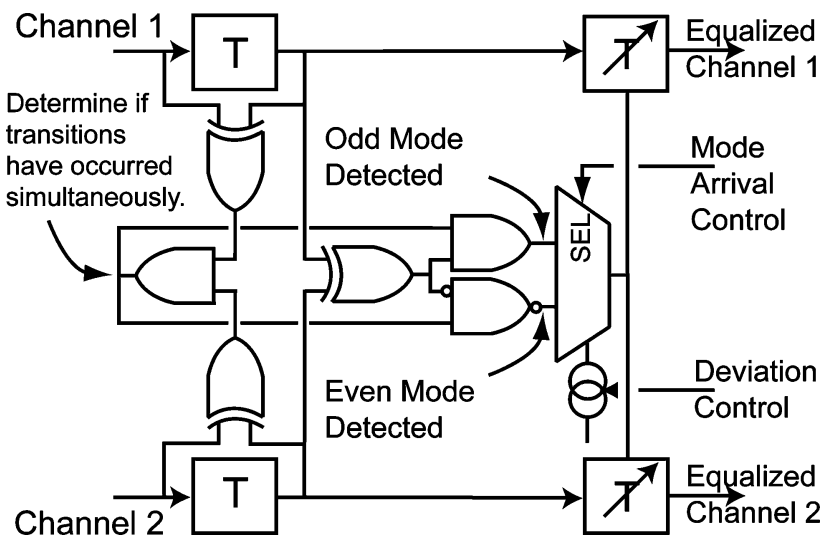

Fig. 12. Implemented version of two-channel CIJ equalizer.

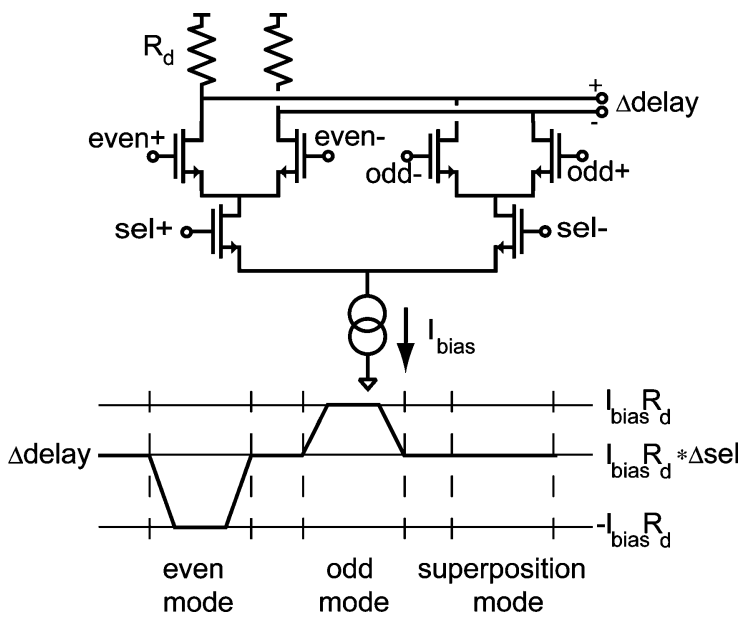

Fig. 13. Schematic and operation of the mode multiplexer.

the select input. When either the even or odd modes occur, the output is driven between + or $-I_{\mathrm{bias}} R_{d}$, respectively. Consequently, control over the total range of arrival times for the odd and even mode can be controlled with $I_{\text {bias }}$.

While the arrival time of the superposition mode is ideally between the even and odd modes, the discussion of the timing offsets and illustration in Fig. 6 of the relative timing of the odd, superposition, and even modes implies that control over the superposition delay is also useful. Additionally, the nonlinear delay variation described in the following paragraphs suggests that independent control of the superposition mode delay is essential. Consequently, the select input controls the relative position of the superposition timing. Note that if the differential select voltage is zero, the voltages corresponding to the odd and even modes are equally spaced.

The time delay element consists of a simple cross-coupled differential pair through which current can be starved. The schematic for the delay stage is shown in Fig. 14. The cross-coupled differential pair can be analyzed as a negative resistance loading the output drain resistance [22]. The advantage of the cross-coupled delay stage is the constant large-signal swing while providing variation of the small-signal characteristics. Additionally, the time delays are tuned for the appropriate tap delay and bit rate. 


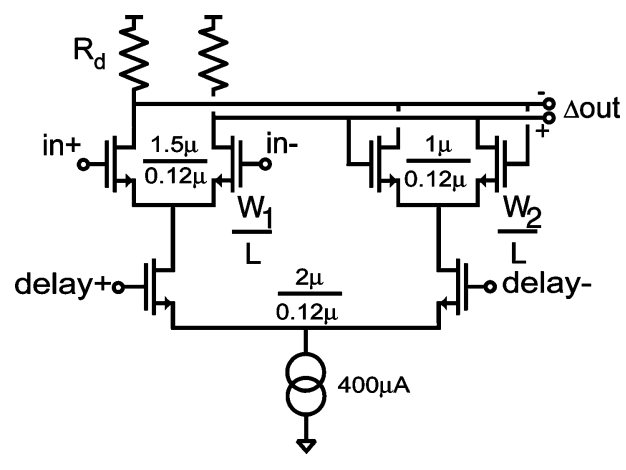

Fig. 14. Schematic of the delay cell.

A first-order analysis demonstrates the delay variation possible in the delay cell. The equivalent circuit for this cell places a negative resistance that depends on the current steered through cross-coupled pair in parallel with $R_{d}$. Consequently, the firstorder transfer function for this stage is

$$
A_{v}(s)=\frac{g_{m 1} R_{d}^{\prime}}{1-g_{m 2} R_{d}^{\prime}} \cdot \frac{1}{1+s \frac{R_{d}^{\prime} C_{d}}{1-g_{m 2} R_{d}^{\prime}}}
$$

where $R_{d}^{\prime}=R_{d}\left\|r_{o 1}\right\| r_{o 2}$. This implies that the delay of the stage is found from the time constant associated with the pole [23]:

$$
\tau_{d}=\frac{R_{d}^{\prime} C_{d}}{1-g_{m 2} R_{d}^{\prime}} .
$$

This variation has been plotted against the actual transient simulation in Fig. 15. This plot also demonstrates the proper sizing of the cross-coupled differential pair with respect to the differential pair. In particular, the desired delay variation is chosen from the amount of crosstalk-induced jitter that should be compensated. Consequently, the size of $W_{2}$ is chosen as a ratio to $W_{1}$. The obvious tradeoff for delay variation from the analysis in (16) is the bandwidth for the high-speed signal. It is clear that the circuit provides for at most $30 \mathrm{ps}$ of delay variation. Consequently, the CIJ equalizer can compensate $\tau_{\mathrm{f}}$ of 15 ps.

The important characteristic of the delay variation in Fig. 15 is that it is nonlinear. If the application only required two delay values, the nonlinearity of the delay variation would not be an important design consideration. However, for this equalizer, three specific delays need to be selected. Consequently, if we desire equally spaced variation as described by the pdf in (9), the voltage swing must be unequal. Therefore, the variable control over the superposition time delay in Fig. 13 improves the operation. Other delay elements offer more tuning linearity and might be considered alternatively but achieving high linearity over a wide range is difficult [24].

The entire two-channel circuit consumes $330 \mathrm{~mA}$ from a $2-\mathrm{V}$ supply. The supply was higher than specified for this fabrication technology to adjust for process variations. This includes additional circuits not described in these results but discussed in [16]. The chip area measures $1 \mathrm{~mm} \times 1 \mathrm{~mm}$ The actual area of the CIJ equalizer is $140 \mu \mathrm{m} \times 100 \mu \mathrm{m}$ and consumes $40 \mathrm{~mA}$ from simulation. The remaining current consumption supports the output buffering.

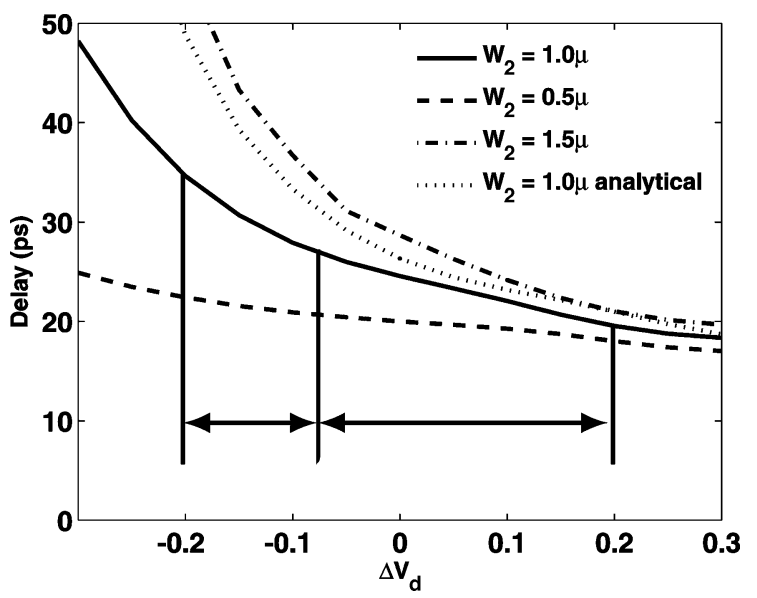

Fig. 15. Transient and analytic delay variation for delay cell illustrated in Fig. 14.

\section{RESULTS}

Two measurements are presented to show the performance improvement of the CIJ equalizer. The first measurement shows a decrease in the rms and peak-to-peak jitter in the data eye. The second measurement is the increase in the timing range that achieves at $10^{-12}$ BER.

The chip is wirebonded to a Rogers 5880 duroid test board on a brass mount. To generate uncorrelated data sequences at 5-10 Gb/s, differential outputs of an Anritsu MP1763C pulse pattern generator (PPG) are delayed with respect to one another by 10 bits. The pseudo-random bit sequences generated by the MP1763C are maximal length sequence, which has low ( $\sim$ zero) autocorrelation after one bit [5].

For this work, the delay value is adjusted manually to reduce the CIJ. It is possible to include adaptation by using a time-to-voltage conversion that samples the timing deviations for particular bit sequences between the neighboring lines.

For 10-Gb/s testing, the two data paths are coupled through $40-\mu \mathrm{m}$-long on-chip transmission lines spaced $400 \mathrm{~nm}$ apart. The coupling capacitance for these lines is $3.34 \mathrm{fF}$ and the coupling is dominated by the mutual capacitance since the two lines are not terminated by the line impedance but instead by the drain resistance. Consequently, the capacitive term in (5) dominates the coupling. These transmission lines are driven with a $400-\mu \mathrm{A}$ buffer and terminated with $4 \mathrm{k} \Omega$. The predicted forward crosstalk coupling time constant is calculated as 6.7 ps. However, the transient simulations indicate that $\tau_{\mathrm{f}}$ is $6 \mathrm{ps}$.

The first measurement collects jitter statistics around the center of the data eye where the jitter is lowest. The rms jitter and peak-to-peak jitter are measured with and without CIJ equalization. The compensation voltage is tuned to provide the minimum jitter. In Fig. 16, the data eye demonstrates that $J_{\sigma}$ reduces from 8.7 to $6.3 \mathrm{ps}$ at $10 \mathrm{~Gb} / \mathrm{s}$. $J_{\mathrm{pp}}$ reduces from 48.9 to 34.2 ps. Using (12), the rms value indicates that the circuit reduced the rms jitter contribution CIJ by 6 ps. The peak-to-peak value indicates that the circuit reduced the peak-to-peak contribution of CIJ by 14.7 ps. From (10) and (11), this implies the $\tau_{f}$ is 7.4 and $8.5 \mathrm{ps}$, respectively. These values are only slightly higher than the $\tau_{f}$ expected from simulation.

For testing at $5 \mathrm{~Gb} / \mathrm{s}$, the two data sequences are coupled through a series combination of the on-chip transmission lines 


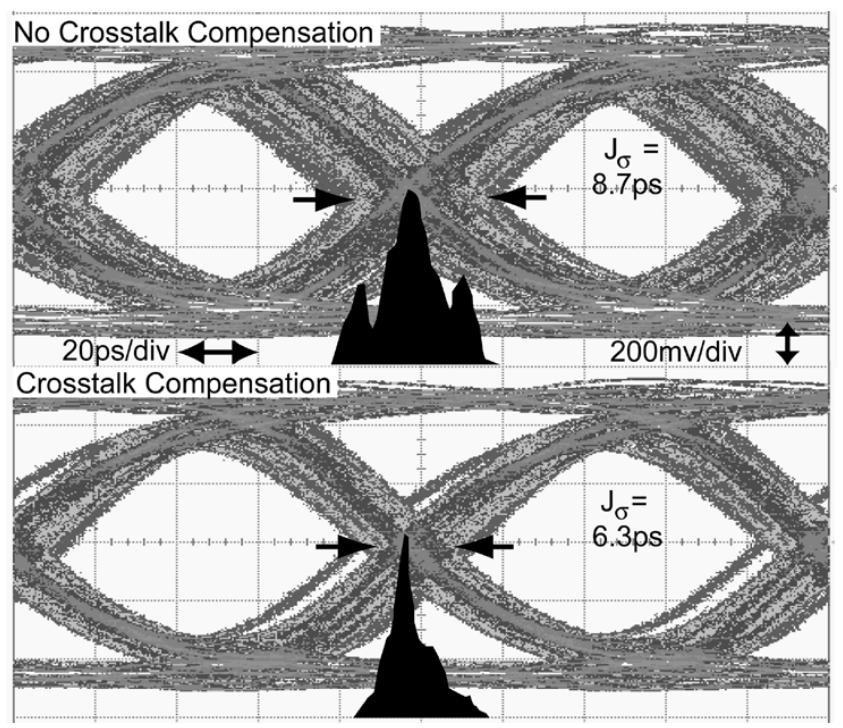

Fig. 16. Data eyes at $10 \mathrm{~Gb} / \mathrm{s}$ before and after equalization.

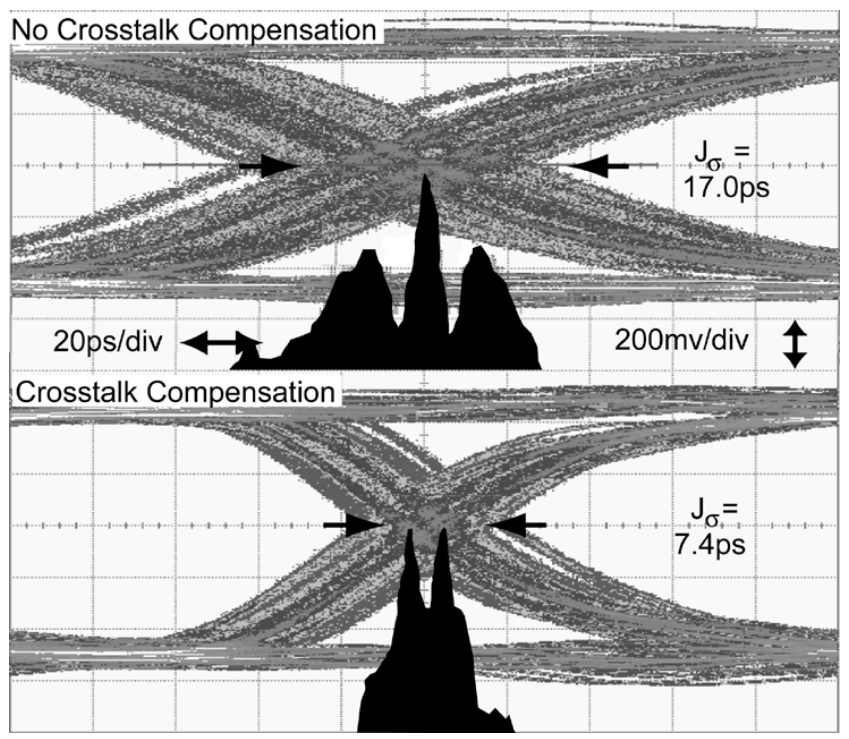

Fig. 17. Data eyes at $5 \mathrm{~Gb} / \mathrm{s}$ before and after equalization.

and coupled microstrip lines fabricated on Rogers 5880 duroid described earlier. A 100-mil line spacing is used for the coupled lines. From Table I, $J_{\sigma, \text { CIJ }}$ is 14.3 ps at the input of the receiver. This contribution overwhelms the impact of the on-chip transmission lines. In Fig. 17, the data eye demonstrates that $J_{\sigma}$ reduces from 17 to $7.4 \mathrm{ps}$ at $5 \mathrm{~Gb} / \mathrm{s}$. Using (12), the rms value indicates that the circuit reduced the rms jitter contribution CIJ by $15.3 \mathrm{ps}$. The peak-to-peak value indicates that the circuit reduced the peak-to-peak contribution of CIJ by 44.7 ps. From (10) and (11), this implies the $\tau_{f}$ is 21.6 and $22.3 \mathrm{ps,} \mathrm{respec-}$ tively. Since the delay variation was nominally limited to $15 \mathrm{ps}$, the CIJ equalization may be further improved. A summary of the jitter statistics at 5 and $10 \mathrm{~Gb} / \mathrm{s}$ is also shown in Table III.

The second measurement sketches the bathtub curve for the eye opening with the Anritsu MP1764C error detector. The BER is recorded at each particular sampling time to form a curve. The notable improvement of $J_{\sigma}$ and $J_{\mathrm{pp}}$ is reflected by the larger eye opening in Figs. 18 and 19 after compensation. A summary of these results at 5 and $10 \mathrm{~Gb} / \mathrm{s}$ is also shown in Table III. To define
TABLE III

IMPROVEMENT OF CROSSTALK-INDUCED JITTER AT 5 AND $10 \mathrm{~Gb} / \mathrm{s}$

\begin{tabular}{|c|c|c|c|}
\hline Equalization & $\mathrm{J}_{\sigma}$ & $\mathrm{J}_{\mathrm{pp}}$ & $10^{-12}$ BER \\
\hline $10 \mathrm{~Gb} / \mathrm{s}$, Before & $8.7 \mathrm{ps}$ & $48.9 \mathrm{ps}$ & $17 \mathrm{ps}$ \\
\hline $10 \mathrm{~Gb} / \mathrm{s}$, After & $6.3 \mathrm{ps}$ & $34.2 \mathrm{ps}$ & $45 \mathrm{ps}$ \\
\hline $5 \mathrm{~Gb} / \mathrm{s}$, Before & $17 \mathrm{ps}$ & $86 \mathrm{ps}$ & $102 \mathrm{ps}$ \\
\hline $5 \mathrm{~Gb} / \mathrm{s}$, After & $7.4 \mathrm{ps}$ & $41.3 \mathrm{ps}$ & $143 \mathrm{ps}$ \\
\hline
\end{tabular}

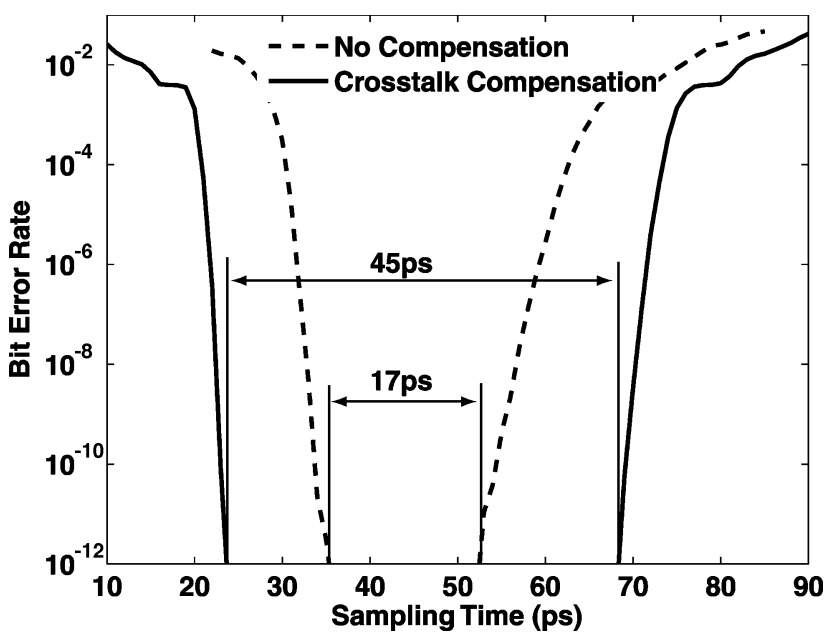

Fig. 18. Bathtub curve resulting before and after equalization at $10 \mathrm{~Gb} / \mathrm{s}$.

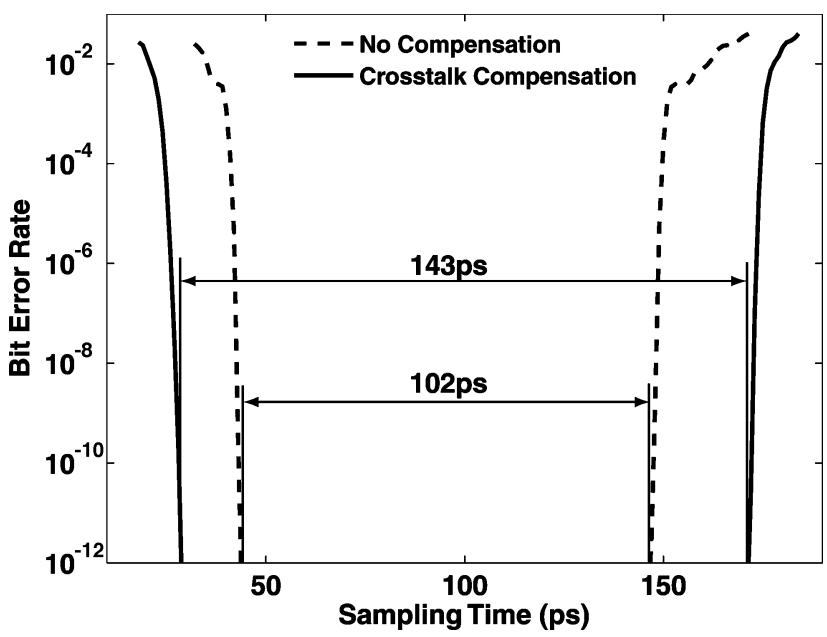

Fig. 19. Bathtub curve resulting before and after equalization at $5 \mathrm{~Gb} / \mathrm{s}$.

a figure for performance of the equalizer, we quote the BER at $10^{-12}$ for the purposes of determining the eye opening. The BER curve measured before compensation shows an opening of 17 ps at $10^{-12}$ BER and 45 ps after compensation at $10 \mathrm{~Gb} / \mathrm{s}$. At $5 \mathrm{~Gb} / \mathrm{s}$, the eye opening increases from 102 to $143 \mathrm{ps}$. This indicates the substantial eye improvement possible due to the CIJ equalizer.

\section{CONCLUSION}

This paper discusses the effect of crosstalk on timing jitter in high-speed serial communications. The analysis demonstrates a method to quantify the amount of crosstalk-induced jitter from the mutual capacitance and inductance between two adjacent 
lines. These coupling components are demonstrated to cause a variation in the time of flight of even and odd transition modes between the adjacent lines. An expression for the total crosstalk-induced jitter is derived and demonstrated on both 2-PAM and 4-PAM signaling schemes. The analysis suggests equalization that compares the transition mode between neighboring lines. The circuit is fabricated in 130-nm MOSFET technology and operates between 5 and $10 \mathrm{~Gb} / \mathrm{s}$. Reduction in the rms and peak-to-peak jitter are demonstrated in the data eye. Bathtub curve measurements verify the improvement of through increase sampling range.

\section{APPENDIX A}

If we assume weak coupling between the lines, we can ignore the impact of secondary reflections (i.e., $\Gamma^{2} \sim 0$ ). In this case, the even and odd mode voltages are delayed versions of the generator voltage. The superposition mode between lines 1 and 2 is

$$
v_{1(2)}(l, t)=\frac{1}{2}\left[V\left(t-T_{e}\right) \pm V\left(t-T_{o}\right)\right]
$$

Therefore, the voltage on line 2 is zero if the two modes propagate at the same speed. In inhomogeneous lines, they do not and the result is a voltage spike on the line. The qualitative upshot of the weak coupling argument is that this spike does not affect the line 1 significantly.

The time of arrival for the superposition mode is thus expressed as $T=L \sqrt{L_{s} C_{s}-L_{m} C_{m}} \sim L \sqrt{L_{s} C_{s}}$.

$$
T_{e(o)} \approx T \sqrt{1 \pm l\left(\frac{L_{m}}{L s}-\frac{C_{m}}{C_{s}}\right)}
$$

Using the binomial expansion for the square root, this equation reduces to

$$
T_{e(o)} \approx T \pm \frac{l}{2}\left(\frac{L_{m}}{Z_{o}}-C_{m} Z_{o}\right)
$$

Analytical results for strong coupling are difficult to reach. Furthermore, strong coupling is problematic for binary data communications since the waveforms are distorted greatly during transmission.

Coupling severely distorts the different waveforms when the arrival times of the even and odd mode become widely separated. When the odd mode occurs well before the even mode, for instance, the superposition mode does not interpolate smoothly between these two modes. Consequently, the transition of the superposition mode is no longer monotonic.

Therefore, a useful limit on strong coupling relates the transition time of the waveform to the amount of coupling. To ensure that the even and odd edges begin and end in the same time interval

$$
t_{r}<2 \tau_{f}
$$

where $t_{r}$ is the rise time (or fall time, whichever is longer) of the signal.

\section{APPENDIX B}

To calculate the threshold crossing time deviation due to crosstalk, $t_{x}$, we can apply a first-order Taylor series to the victim and aggressor signal using the original result for an unaffected signal. This is reasonable since often the rising edge is monotonic. The victim signal is approximated as

$$
r_{1}\left(t_{x}\right)=\sum_{n=-\infty}^{0} a_{n}\left(g\left(t_{c}-n T\right)+\left(t_{x}-t_{c}\right) g^{\prime}\left(t_{c}-n T\right)\right) \text {. }
$$

In this expression, $g^{\prime}(t)$ is the first derivative of the pulse response. The first term in this equation represents the pulse response without the aggressor signal. Therefore, (7) becomes

$$
\sum_{n=-\infty}^{0} a_{n}\left(t_{x}-t_{c}\right) g^{\prime}\left(t_{c}-n T\right)+\tau_{f} b_{n} g^{\prime}\left(t_{x}-n T\right)=0 .
$$

Unfortunately, the argument of the derivative of the pulse response contains different times. To handle this, we make a Taylor series approximation for $g^{\prime}\left(t_{x}-n T\right)$. Consequently, we have a closed-form solution for $t_{x}$ :

$$
t_{x}=t_{c}-\tau_{f} \frac{\sum_{n=-\infty}^{0} b_{n} g^{\prime}\left(t_{c}-n T\right)}{\sum_{n=-\infty}^{0}\left(a_{n} g^{\prime}\left(t_{c}-n T\right)+\tau_{f} b_{n} g^{\prime \prime}\left(t_{c}-n T\right)\right)} .
$$

Two approximations are particularly relevant. First, the numerator and denominator both contain the derivative of the pulse response. Typically, this derivative is strongest at the rising and falling edges $\left(t_{c}=0\right.$ and $\left.T\right)$, respectively. Consequently, all other terms in the summation can be assumed to be zero. Additionally, the solution simplifies further if we assume both the rising and falling edges have the are equal and opposite transitions, i.e., $g^{\prime}\left(t_{c}\right)=-g^{\prime}\left(t_{c}+T\right)$. Now

$$
t_{x}=t_{c}-\tau_{f} \frac{b_{0}-b_{-1}}{a_{0}-a_{-1}+\tau_{f} \frac{g^{\prime \prime}\left(t_{c}\right)}{g^{\prime}\left(t_{c}\right)}\left(b_{0}-b_{-1}\right)} .
$$

Finally, we can make the assumption that the second derivative of the slope can be taken to be zero near $t_{c}$. The threshold crossing time in the presence of the aggressor can be simply expressed as

$$
t_{x}=t_{c}-\tau_{f} \cdot \frac{b_{o}-b_{-1}}{a_{o}-a_{-1}} .
$$

The crosstalk-induced jitter is defined as

$$
t_{c, \mathrm{CIJ}}=-\tau_{f} \cdot \frac{b_{0}-b_{-1}}{a_{0}-a_{-1}} .
$$

\section{APPENDIX C}

Relating measurements of the jitter in 4-PAM to the BER is not straightforward. Because of the multiple thresholds required to detect a four level signal, there are many transitions in the 4-PAM data eye. For detecting any one symbol, only a subset of these transitions is important. These issues are discussed in [25]. 
In particular, we use a definition for the jitter that is straightforward from a measurement perspective. Locating the histogram around the center of the middle transition captures many, but not all of the transitions that occur. A pdf describing the CIJ can be constructed for analyzing the jitter statistics for a histogram located at the middle transition crossing (i.e., $0 \mathrm{~V}$ ):

$$
\begin{aligned}
\operatorname{pdf}_{\mathrm{CIJ}}\left(t_{c}\right)= & \frac{1}{64} \delta\left(t_{c}-3 \tau_{f}\right)+\frac{1}{32} \delta\left(t_{c}-2 \tau_{f}\right) \\
& +\frac{1}{32} \delta\left(t_{c}+\frac{3 \tau_{f}}{2}\right)+\frac{1}{8} \delta\left(t_{c}+\tau_{f}\right) \\
& +\frac{1}{32} \delta\left(t_{c}+\frac{2 \tau_{f}}{3}\right)+\frac{3}{32} \delta\left(t_{c}+\frac{\tau_{f}}{2}\right) \\
& +\frac{3}{64} \delta\left(t_{c}+\frac{\tau_{f}}{3}\right)+\frac{1}{4} \delta\left(t_{c}\right)+\frac{3}{64} \delta\left(t_{c}-\frac{\tau_{f}}{3}\right) \\
& +\frac{3}{32} \delta\left(t_{c}-\frac{\tau_{f}}{2}\right)+\frac{1}{32} \delta\left(t_{c}-\frac{2 \tau_{f}}{3}\right) \\
& +\frac{1}{8} \delta\left(t_{c}-\tau_{f}\right)+\frac{1}{32} \delta\left(t_{c}-\frac{3 \tau_{f}}{2}\right) \\
& +\frac{1}{32} \delta\left(t_{c}-2 \tau_{f}\right)+\frac{1}{64} \delta\left(t_{c}-3 \tau_{f}\right) .
\end{aligned}
$$

In the absence of noise, expressions for the rms and peak-topeak jitter for CIJ can be calculated as for 2-PAM. The standard deviation of the CIJ for 4-PAM, $J_{\sigma, \mathrm{CIJ}}$, is determined from the pdf in (28):

$$
J_{\sigma, \mathrm{CIJ}}=\tau_{f} \sqrt{\frac{145}{144}} \approx \tau_{f}
$$

\section{ACKNOWLEDGMENT}

The authors would like to thank B. Analui at Caltech for helpful discussions. Foundry access was provided by IBM Research at Yorktown Heights, NY. Additionally, the authors thank the Rogers Corporation for the generous donation of duroid.

\section{REFERENCES}

[1] R. Kollipara et al., "Design, modeling, and characterization of highspeed backplane interconnects," presented at the DesignCon 2003, [Online.] Available: http://www.rambus.com/news/technical_docs/designcon2003_paper.pdf.

[2] V. Stojanovic and M. Horowitz, "Model and analysis of high-speed serial links," in Proc. IEEE Conf. Custom Integrated Circuits, Sep. 2003, pp. 589-594.

[3] J. Stonick et al., "An adaptive PAM-4 5-Gb/s backplane transceiver in 0.25 um CMOS," IEEE J. Solid-State Circuits, vol. 38, no. 3, pp. 436-443, Mar. 2003

[4] V. Stojanovic et al., "Autonomous dual-mode (PAM2/4) serial link transceiver with adaptive equalization and data recovery," IEEE J. Solid-State Circuits, vol. 40, no. 4, pp. 1012-1026, Apr. 2005.

[5] J. Proakis, Digital Communications. New York: McGraw-Hill, 2001.

[6] W. Dally and J. Poulton, Digital Systems Engineering. Cambridge, U.K.: Cambridge Univ. Press, 1998.

[7] A. Deutsch, "Electrical characteristics of interconnections for highperformance systems," Proc. IEEE, vol. 86, no. 2, pp. 315-357, Feb. 1998

[8] J. Zerbe et al., "A $2 \mathrm{~Gb} / \mathrm{s} /$ pin 4-PAM parallel bus interface with transmit crosstalk cancellation, equalization, and integrating receivers," in IEEE Int. Solid-State Circuits Conf. Dig. Tech. Papers, Feb. 2001, pp. 66-67.

[9] C. Pelard et al., "Realization of multigigabit channel equalization and crosstalk cancellation integrated circuits," IEEE J. Solid-State Circuits, vol. 39, no. 10, pp. 1659-1669, Oct. 2004.

[10] Y. Hur et al., "Equalization and near-end crosstalk (NEXT) noise cancellation for $20 \mathrm{~Gb} / \mathrm{s}$ 4-PAM backplane serial I/O interconnects," IEEE T. Microwave Theory Tech., vol. 53, no. 1, pp. 246-255, Jan. 2005.

[11] R. Ho, K. Mai, and M. Horowitz, "The future of wires," Proc. IEEE, vol. 89, no. 4, pp. 490-504, Apr. 2001.

[12] J. Buckwalter, B. Analui, and A. Hajimiri, "Data-dependent jitter and crosstalk-induced bounded uncorrelated jitter in copper interconnects," in IEEE Int. Microwave Symp. Dig., Jun. 2004, pp. 1627-1630.

[13] "Fiber Channel-Methodologies for jitter and signal quality specification (MJSQ)," Int. Committee for Information Technology Standardization (INCITS), Tech. Rep. Rev. 10.0, Mar. 10, 2003.

[14] A. Kuo et al., "Jitter models and measurement methods," in Proc. IEEE Int. Test Conf., 2004, pp. 1295-1302.

[15] M. Li and J. Wilstrup, "Paradigm shift for jitter and noise in design and test $>\mathrm{Gb} / \mathrm{s}$ communication systems," in Proc. IEEE Int. Conf. Computer Design, Oct. 2003, pp. 467-472.

[16] J. Buckwalter and A. Hajimiri, "Crosstalk-induced jitter equalization," in Proc. IEEE Custom Integrated Circuits Conf., Sep. 2005, pp. 402-405.

[17] D. Pozar, Microwave Engineering. Reading, MA: Addison-Wesley, 1990.

[18] S. J. Orfinidas. Electromagnetic waves and antennas. [Online]. Available: http://www.ece.rutgers.edu/ orfanidi/ewa/

[19] Y.-S. Sohn et al., "Empirical equations for electrical parameters of coupled microstrip lines with one side exposed to air," Electron. Lett., vol. 35 , no. 11 , pp. 906-907.

[20] - "Empirical equations for electrical parameters of coupled microstrip lines for crosstalk estimation in printed circuit boards," IEEE Trans. Adv. Packag., vol. 24, no. 4, pp. 521-527, Nov. 2001.

[21] R. Farjad-Rad, C.-K. K. Yang, and M. A. Horowitz, "A $03 \mu \mathrm{m}$ CMOS 8-Gb/s 4-PAM serial link transceiver," IEEE J. Solid-State Circuits, vol 35, no. 5, pp. 757-764, May 2000.

[22] B. Razavi, Design of Analog CMOS Integrated Circuits. New York: McGraw-Hill, 2001.

[23] T. Lee, The Design of CMOS Radio-Frequency Integrated Circuits. Cambridge, U.K.: Cambridge Univ. Press, 1998.

[24] J. Maneatis, "Low-jitter process-independent DLL and PLL based on self-biased techniques," IEEE J. Solid-State Circuits, vol. 31, no. 11, pp. 1723-1732, Nov. 1996.

[25] J. Buckwalter, "Deterministic jitter in broadband communication," Ph.D. dissertation, California Inst. Technol., Pasadena, 2006.

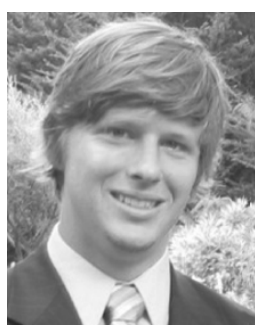

James F. Buckwalter ( $\left.\mathrm{S}^{\prime} 01\right)$ received the B.S. degree in electrical engineering from the California Institute of Technology (Caltech), Pasadena, in 1999. He received the M.S. degree in electrical engineering in 2001 from the University of California at Santa Barbara, where he developed coupled phase-locked loops for microwave beam-steering under the supervision of Prof. Robert York. He returned to Caltech to complete the Ph.D. degree with Prof. Ali Hajimiri in January 2006. His thesis focused on problems relating to timing jitter in high-speed communications and testing.

He was a Research Scientist at Telcordia Technologies from 1999 to 2000 where he worked on rate-agile burst-mode electronics under a Next-Generation Internet DARPA project. He worked at IBM T. J. Watson Research Center, Yorktown Heights, NY, during the summer of 2004.

Dr. Buckwalter was awarded an IBM Ph.D. fellowship in 2004. 


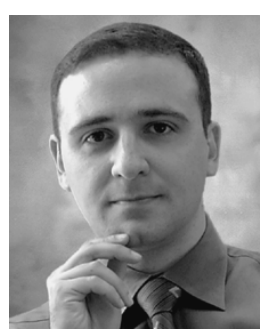

Ali Hajimiri (S'95-M'99) received the B.S. degree in electronics engineering from the Sharif University of Technology, Tehran, Iran, and the M.S. and Ph.D. degrees in electrical engineering from the Stanford University, Stanford, CA, in 1996 and 1998, respectively.

He was a Design Engineer with Philips Semiconductors, where he worked on a BiCMOS chipset for GSM and cellular units from 1993 to 1994. In 1995, he was with Sun Microsystems, where he worked on the UltraSPARC microprocessor's cache RAM design methodology. During the summer of 1997, he was with Lucent Technologies (Bell Labs), Murray Hill, NJ, where he investigated low-phase-noise integrated oscillators. In 1998, he joined the Faculty of the California Institute of Technology, Pasadena, where he is an Associate Professor of electrical engineering and the Director of the Microelectronics Laboratory. He is the author of The Design of Low Noise Oscillators (Kluwer, 1999) and holds several U.S. and European patents. He is a cofounder of Axiom Microdevices Inc. His research interests are high-speed and RF integrated circuits.

Dr. Hajimiri is an Associate Editor of the IEEE JOURNAL OF SOLID-STATE CIRCUITS (JSSC) and a member of the Technical Program Committee of the IEEE International Solid-State Circuits Conference (ISSCC). He has also served as an Associate Editor of IEEE TRANSACTIONS ON CIRCUITS AND SYSTEMS-PART II, a member of the Technical Program Committees of the International Conference on Computer Aided Design (ICCAD), Guest Editor of the IEEE TRANSACTIONS ON MicROWAVE THEORY AND TECHNIQUES, and the Guest Editorial Board of Transactions of Institute of Electronics, Information and Communication Engineers of Japan (IEICE). He was selected to the top 100 innovators (TR100) list in 2004 and is a Fellow of the Okawa Foundation. $\mathrm{He}$ is a recipient of the Teaching and Mentoring Award at Caltech. He was the Gold Medal winner of the National Physics Competition and the Bronze Medal winner of the 21st International Physics Olympiad, Groningen, The Netherlands. He was a co-recipient of the ISSCC 1998 Jack Kilby Outstanding Paper Award, two times co-recipient of CICC's Best Paper Awards, and a three times winner of the IBM faculty partnership award as well as the National Science Foundation CAREER Award. 